\title{
First reports of serious adverse drug reactions in recent weeks
}

The following table contains an overview of first published case reports of serious adverse drug reactions identified in the international literature in recent weeks by the drug safety alerting service Reactions Weekly. An event is serious (US FDA MedWatch definition) when the patient outcome is death, life threatening, hospitalization, disability, congenital anomaly or requires intervention to prevent permanent impairment or damage. Reactions and the customized Reactions Pharmacovigilance Service are produced by Adis, and monitor $>4000$ journals, including relevant MEDLINE- and EMBASE-indexed journals, as well as companion journal supplements, major scientific meetings, the newsletters from the $>80$ national centres participating in the WHO International Drug Monitoring Programme, media releases, pharmaceutical company websites, and regulatory agency websites.

\author{
Drug and serious adverse reaction \\ Aceclofenac: acute tubulointerstitial nephritis \\ Agomelatine: delirium \\ Baclofen: increased blood triglyceride levels \\ Ceftaroline fosamil: agranulocytosis in an elderly patient \\ Colistin: contact tracheobronchitis \\ Insulin detemir: interactions with amoxicillin, clarithromycin and \\ omeprazole leading to hypoglycaemia in an elderly patient \\ Linagliptin/vildagliptin: hepatitis in an elderly patient \\ Phenylephrine: type IV reaction manifesting as drug \\ hypersensitivity syndrome \\ Tocilizumab: hepatitis \\ Tocilizumab: mesangial proliferative glomerulonephritis \\ Valproate: panhypogammaglobulinaemia in an infant \\ Vemurafenib: pancreatitis
}

\section{Reference}

Gupta M, Cruz SD, Nada R, et al. Aceclofenac-induced acute tubulointerstitial nephritis in a patient with diabetes. BMJ Case Rep. 2013; pii:bcr2013009217.

Viejo Sacha MG, De Ambrosi B, Muñoz C, et al. Two cases of delirium with agomelatine therapy. Ann Clin Psychiatry. 2013;25(1):67-8.

Clarisse H, Imbert B, Belzeaux R, et al. Baclofen and risperidone association increases dramatically triglycerides level. Alcohol Alcohol. 2013;48(4):515-6.

Rimawi RH, Frenkel A, Cook PP. Ceftaroline: a cause for neutropenia. J Clin Pharm Ther. 2013;38(4):330-2.

Vogel S, Varga R, Friedl T, et al. Respiratory insufficiency due to contact allergy to colistin [abstract no. 808]. Allergy. 2012;67 Suppl S96:313-4.

Otsuka SH. Severe hypoglycemia from Helicobacter pylori triple-drug therapy and insulin detemir drug interaction. Pharmacotherapy. 2013;33(4):e45-9.

Danish Health and Medicines Authority. Acute pancreatitis and hepatitis associated with the use of vildagliptin $\left(\right.$ Galvus $\left.{ }^{\circledR}\right)$ and linagliptin (Trajenta ${ }^{\circledR}$ ) reported as potential adverse reactions. Dan Pharmacovig Update. 2013;4(6):5.

Rice SA, Swale VJ, Haque R, et al. Erythroderma in the emergency department. BMJ. 2013;346:f3613.

Alfreijat M, Habibi M, Bhatia P, et al. Severe hepatitis associated with tocilizumab in a patient with rheumatoid arthritis. Rheumatology (Oxford). 2013;52(7):1340-1.

Matsuo Y, Mizoguchi F, Kohsaka H, et al. Tocilizumab-induced immune complex glomerulonephritis in a patient with rheumatoid arthritis. Rheumatology (Oxford). 2013;52(7):1341-3.

Eom TH, Lee HS, Jang PS, et al. Valproate-induced panhypogammaglobulinemia. Neurol Sci. 2013;34(6):1003-4.

Muluneh B, Buie LW, Collichio F. Vemurafenib-associated pancreatitis: case report. Pharmacotherapy. 2013;33(4):e43-4. 